\title{
BMJ Open Protocol for developing, disseminating and implementing a core outcome set for endometriosis
}

To cite: Hirsch M, Duffy JMN, Barker C, et al. Protocol for developing, disseminating and implementing a core outcome set for endometriosis. BMJ Open 2016;6:e013998. doi:10.1136/bmjopen-2016013998

- Prepublication history and additional material is available. To view please visit the journal (http://dx.doi.org/ 10.1136/bmjopen-2016013998).

Received 23 August 2016 Revised 21 October 2016 Accepted 24 October 2016

CrossMark

For numbered affiliations see end of article.

Correspondence to Dr Martin Hirsch; m.hirsch@qmul.ac.uk

\section{ABSTRACT}

Introduction: Endometriosis is a common gynaecological disease characterised by pain and subfertility. Randomised controlled trials evaluating treatments for endometriosis have reported many different outcomes and outcome measures. This variation restricts effective data synthesis limiting the usefulness of research to inform clinical practice. To address these methodological concerns, we aim to develop, disseminate and implement a core outcome set for endometriosis engaging with key stakeholders, including healthcare professionals, researchers and women with endometriosis.

Methods and analysis: An international steering group has been established, including healthcare professionals, researchers and patient representatives. Potential outcomes identified from a systematic review of the literature will be entered into a modified Delphi method. Key stakeholders will be invited to participate including healthcare professionals, researchers and women with endometriosis. Participants will be invited to score individual outcomes on a nine-point Likert scale anchored between 1 (not important) and 9 (critical). Repeated reflection and rescoring should promote whole and individual stakeholder group converge towards consensus, 'core', outcomes. Highquality outcome measures will be associated with core outcomes.

Ethics and dissemination: The implementation of a core outcome set for endometriosis within future clinical trials, systematic reviews and clinical guidelines will enhance the availability of comparable data to facilitate evidence-based patient care. This study was prospectively registered with Core Outcome Measures in Effectiveness Trials Initiative; number: 691.

\section{INTRODUCTION}

Endometriosis is a chronic inflammatory disease characterised by lesions of endometrial-like tissue outside the uterus that is associated with pelvic pain and/or infertility. ${ }^{1}$ It is a common condition affecting women of reproductive age and may be associated with substantially reduced quality of life. ${ }^{2}$ To address this considerable health burden novel treatments are continually being developed, which require robust evaluation. While significant effort has been paid to developing randomised controlled trial methods, the collection and reporting of outcomes and outcomes measures has been largely overlooked. The consequence of this is a plethora of differing outcomes that make drawing conclusions across a group of studies difficult and, sometimes, impossible.

We performed a systematic review of randomised trials evaluating treatments for endometriosis. ${ }^{3}$ A total of 54 trials reported 164 different outcomes measured by 113 different definitions and instruments. The commonest pain outcome, dysmenorrhoea, was measured by ten different instruments. The commonest fertility outcome, pregnancy, was measured by three different definitions. The lack of consensus regarding the collection and reporting of outcomes prohibits the comparison and combination of individual trial data, limiting the usefulness of research to inform clinical practice.

The endometriosis research community has previously engaged with standardising important aspects of research design. The Art and Science of Endometriosis meeting, convened by the National Institutes of Health, has published recommendations regarding the standardisation of research design in several areas including entry criteria and outcome measures for pain symptoms. ${ }^{4}$ The World Endometriosis Research Foundation Endometriosis Phenome and Biobanking Harmonization Project (WERF EPHect) has published tools for the standardisation of research design in several areas including clinical, covariate and surgical 
phenotype recording and specimen collection, processing and storage. ${ }^{5-8}$ Their work continues, involving global participants from a range of stakeholder groups including healthcare professionals, researchers, industry representatives and women with endometriosis, reflecting the enthusiasm of our specialty to work together to improve research design and clinical care.

The next challenge is to address the unwarranted, unhelpful and often confusing variation in outcome collection and reporting. The development and use of a core outcome set would help to address this challenge. Core outcome sets are well-defined, discriminatory and feasible outcomes routinely collected and reported in randomised trials and systematic reviews. They represent a minimum data set of outcomes selected and prioritised by key stakeholders including healthcare professionals, researchers and women with endometriosis. ${ }^{9}$ The development and use of a core outcome set does not enforce harmony at the expense of innovation. The existence or use of a core outcome set does not imply that outcomes in an endometriosis trial should be restricted. ${ }^{9}$ Rather, there is an expectation that the core outcomes will be collected and reported, making it easier for the results of trials to be compared, contrasted and combined as appropriate; while researchers continue to explore other outcomes as well. ${ }^{10} 11$

Recognising that the current inconsistency in outcome reporting is a serious hindrance to progress in our specialty, 80 editors of Women's Health journals have formed a consortium to support the development, dissemination and implementation of core outcome sets. ${ }^{10}$ The Core Outcomes in Women's and Newborn Health (CROWN) initiative (http://www.crown-initiative.org) will support the dissemination and implementation of a core outcome set for endometriosis to increase the value of an initial research effort and ensure all future endometriosis trials report core outcomes and, therefore, routinely contribute data to important research questions.

Other specialties have succeeded in developing core outcome sets. An international initiative has developed a core outcome set for randomised trials evaluating interventions for chronic pain. The Initiative on Methods, Measurement, and Pain Assessment in Clinical Trials (IMMPACT) has developed a core outcome set including six core outcome domains: (1) pain, (2) physical functioning, (3) emotional functioning, (4) participant ratings of improvement and satisfaction with treatment, (5) symptoms and adverse events and (6) participant disposition. ${ }^{12}$

\section{Objective}

We aim to produce, disseminate and implement a core outcome set for endometriosis.

\section{METHODS AND ANALYSIS}

Prospective registration

This study has been prospectively registered with the Core Outcome Measures in Effectiveness Trials
(COMET) initiative, the registration number is 691 and is available online (http://www.comet-initiative.org/ studies/details/691).

\section{Steering group}

An international steering group, including healthcare professionals, researchers and women with endometriosis, has been formed to guide the development of this core outcome set.

\section{Scope of this core outcome set}

The steering group has recommended the core outcome set should apply to clinical studies evaluating therapeutic interventions for women with endometriosis and follow established core outcome set development methodology (figure 1). All therapeutic interventions for endometriosis will be considered regardless of type, setting or mode of administration. We are not seeking to reach consensus regarding the standardisation of study design including other clinical, covariate and surgical phenotype recording nor specimen collection, processing and storage. The authors acknowledge the established tools in these areas. ${ }^{5-8}$

\section{Step 1: identifying potential outcomes}

We performed a systematic review of randomised trials evaluating therapeutic interventions for treatment of endometriosis. ${ }^{3}$ We have extracted all outcomes and outcome measures reported within the trial reports. Working with patient and public representatives, we have developed lay definitions for these outcomes. The outcomes will be arranged into five domains: pain, subfertility, quality of life, harm and resource utilisation which, following the steering group's agreement, will be entered into a modified Delphi method.

\section{Step 2: determining core outcomes}

The core outcomes will be determined using a modified Delphi method. The method consists of a series of controlled rounds, where repeated surveys are administered. $^{13}$ The modified Delphi method facilitates repeated reflection and rescoring. This promotes whole and individual stakeholder group convergence on a consensus of 'core' outcomes and has advantages over less structured consensus methods. An online-modified Delphi method allows for scoring without the influence of dominant individuals or junior participants feeling obliged to agree with more senior members. Web-based Delphi surveys facilitate international participation and are considered feasible, efficient and acceptable to the user. $^{13}{ }^{14}$ The modified Delphi method will be delivered within a web-based software hosted, designed and delivered by the University of Liverpool.

All key stakeholders will be invited to participate including gynaecologists managing pain or subfertility associated with endometriosis, chronic pain experts, health psychologists, family physicians, researchers and women with endometriosis. There are no clear recommendations 
Figure 1 Study outline. This flow chart outlines the methods of core outcome set formation.

\begin{tabular}{|c|c|}
\hline \multicolumn{2}{|c|}{ Identifying Potential Outcomes } \\
\hline Systematic Review & What outcomes have been reported before? \\
\hline \multicolumn{2}{|c|}{ Determining Core Outcomes } \\
\hline Modified Delphi Method & Combining professional and patients' views \\
\hline Consensus meeting & Stakeholder consultation \\
\hline \multicolumn{2}{|c|}{ Stage 3 Determining How Core Outcomes Should Be Measured } \\
\hline Quality Assessment & Ensuring outcome measures fit for purpose \\
\hline Stakeholder Consultation & Final consensus \\
\hline Cor & mes Set for Endometriosis \\
\hline
\end{tabular}

for calculating the required sample size; based on previous studies, we will aim to include a minimum of 18 participants from each stakeholder group. ${ }^{13}$

\section{Delphi survey pilot}

The Delphi survey will be developed to ensure the ease of completion using appropriate terminology and phrasing. The Delphi survey will be piloted by the study committee and a sample of stakeholders before it is accessible to all stakeholders.

\section{Round 1}

Participants will be asked to register online, provide demographic details and commit to both rounds (box 1). They will be allocated a unique identifier, which will anonymise their responses. Outcomes will be listed in five domains. Outcomes within each domain will be listed alphabetically, and participants will be asked to score individual outcomes using a nine-point Likert Scale anchored between 1 (not important) to 9 (critical). This scale was created by the Grading of Recommendations Assessment, Development and Evaluation (GRADE) working group, and it has been widely adopted by core outcome set developers. ${ }^{15}$ During the first round, participants will be invited to

Box 1 How do I contribute to improving endometriosis research?

The authors acknowledge the expertise and commitment of this journal's readership to improving patient care. They warmly invite readers to participate in the modified Delphi survey by registering their interest to participate here: http://www.eepurl.com/bNC081 suggest additional outcomes. The round will close following a 4-week window.

For each outcome, the median and IQR of scores will be calculated and summarised graphically for the whole and individual stakeholder group responses using DelphiManager. Additional outcomes listed by participants will be reviewed by the steering committee and, if novel, listed in round 2.

\section{Round 2}

Participants will be presented with individual stakeholder group response and asked to reflect on the similarities and differences observed before proceeding to score each outcome again. Additional outcomes proposed in round 1 of the Delphi survey will be added and scored once without reflection. The round will close following a 4-week window.

For each outcome, the median and IQR of scores will be summarised graphically by whole and individual stakeholder group response. A standardised definition of this round's results will enable individual outcomes to be classified:

1. Consensus in (classify as a core outcome): over $70 \%$ of participants in each stakeholder group score this outcome domain 'critical' AND $<15 \%$ of participants in each stakeholder group score outcome domain 'not important'.

2. Consensus out (do not classify as a core outcome): over $70 \%$ of participants in each stakeholder group score outcome domain 'not important' AND $<15 \%$ of participants in each stakeholder group score outcome domain 'critical'.

3. No consensus (do not classify as a core outcome): anything else. $^{13}$ 
In the unlikely event that there are more than ten core outcomes in a single domain the steering group will be able to alter the threshold for classification of consensus in. Round 2 results will be reviewed by the steering group to consider whether there is a need for a further Delphi survey round.

\section{Step 3: stakeholder consultation}

This final phase will involve a face-to-face meeting with key stakeholders. The meeting will include a range of views from participants that will be purposefully sampled from those who have completed all rounds of the Delphi study. The objective of the consensus meeting will be to discuss no consensus outcomes and a final core outcome set for endometriosis. A meeting is planned where the results from each round of the Delphi survey will be presented. To ensure unbiased consensus formation among a group of varied participants, the steering committee will ensure that the meeting is informal, inclusive, participatory and values all opinions. ${ }^{14}$ To facilitate dissemination and implementation, we will invite editors from key journals, for example the British Medical Journal, and funders of endometriosis research.

\section{Step 4: measuring core outcomes}

Once core outcomes are agreed on, it will be important to determine how the outcomes should be measured. ${ }^{16}{ }^{17}$ Potential outcome measurement instruments will be evaluated following a consensus-based guideline for the selection of outcome measurement instruments for outcomes included in a core outcome set. ${ }^{17}$ This involves a four-step process for the identification of outcome measurement instruments for an established set of core outcomes: (1) conceptual considerations; (2) finding existing outcome measurement instruments; (3) quality assessment of outcome measurement instruments and (4) generic recommendations for the selection of outcome measurement instruments for a core outcome set. This approach will ensure, for example, that all core outcomes will still be included in the highly unlikely event that all previous endometriosis studies failed to include a particular core outcome measure.

High-quality outcome measures will be associated with each core outcome. Where multiple high-quality instruments exist, priority will be given to instruments used within the WERF EPHect tools or core outcome measures for chronic pain clinical trials: IMMPACT recommendations. $^{4-8}{ }^{12}$ If no high quality outcome instruments exist for a core outcome, this will be acknowledged.

\section{ETHICS AND DISSEMINATION \\ Ethical review}

We asked the advice of the National Research Ethics Service (NRES) about whether this study required ethical review by an NHS Research Ethics Committee, and they advised that this should be considered as service evaluation and development (see online supplementary appendix 1). All participants involved will be asked for their consent before participation in the Delphi study, and all procedures will be conducted according to the Declaration of Helsinki.

\section{Dissemination}

Implementing and disseminating a core outcome set for endometriosis in future clinical studies, systematic reviews and clinical guidelines could make a profound contribution to advancing the reach and relevance of research to inform clinical practice, enhance patient care and improve patient outcomes.

The selection of appropriate outcomes and outcome measures in future clinical trials is critical. The development of a core outcome set ensures that consensus outcomes important to all stakeholders, including patients, are routinely collected and reported. The Standard Protocol Items Recommendations for Interventional Trials (SPIRIT) statement recommends the use of core outcome sets where they exist. ${ }^{18}$ An endorsement by national and international funders, including National Institutes of Health, will facilitate (and fund) the collection and reporting of core outcomes.

The CROWN initiative, supported by 80 specialty journals, including the Cochrane Gynaecology and Fertility Group, has resolved to implement core outcome sets. Participating journals will require authors to report the results for core outcomes and offer conclusions based on these outcomes rather than non-core or surrogate outcomes. $^{10}$

The production of high quantity and quality comparable data to be summarised within systematic reviews to inform clinical practice guidelines would be an important step forward for guideline developers. The National Institute of Clinical Excellence encourages the use of core outcomes sets where available when selecting outcomes during evidence scoping and synthesis. A core outcome set for endometriosis could directly influence national and international clinical practice.

\section{CONCLUSION}

The development of a core outcome set in endometriosis will enable the collection and reporting of a minimum data set important to all stakeholders, including women with endometriosis. Harmonising outcome collection and reporting for future clinical trials, systematic reviews and clinical guidelines will make a profound and important contribution to patient care.

\section{Author affiliations}

${ }^{1}$ Women's Health Research Unit, Barts and the London School of Medicine and Dentistry, London, UK

${ }^{2}$ Balliol College, University of Oxford, Oxford, UK

${ }^{3}$ Radcliffe Women's Health Patient Participation Group, University of Oxford, Oxford, UK

${ }^{4}$ World Endometriosis Society, Vancouver, British Columbia, Canada 
${ }^{5}$ World Endometriosis Research Foundation, London, UK

${ }^{6}$ Robinson Research Institute, University of Adelaide, Adelaide, South Australia, Australia

${ }^{7}$ Cochrane Gynaecology and Fertility Group, University of Auckland, Auckland, New Zealand

Acknowledgements The authors thank members of the Radcliffe Women's Health Patient Participation Group. They thank colleagues at the Women's Health Research Unit, Queen Mary, University of London including Tracy Holtham and Rehan Khan for administrative and technical support. They also thank David Mills for administrative and material support.

Funding This study is funded by the Endometriosis Millennium Fund, Royal College of Obstetricians and Gynaecologists. The funder has no role in the design and conduct of the study, the collection, management, analysis, or interpretation of data, or manuscript preparation.

Collaborators International Collaboration to Harmonise Outcomes and Measures for Endometriosis (iHOME).

Contributors MH, JMND and KSK have been involved with the study's conception and design. MH, JMND, CB, LH, BM, NPJ, KSK and CF will be involved in the acquisition, analysis and interpretation of data and will provide administrative, technical or material support. MH, JMND, KSK, NPJ and CF will be involved in the statistical analysis. MH and JMND have drafted the manuscript. All authors have critically revised the manuscript for important intellectual content. All authors have read and approved the manuscript.

Competing interests NPJ has received conference expenses from Bayer Pharma, Merck-Serono and MSD; research funding from AbbVie and has been a consultant to Vifor Pharma. The remaining authors report no competing interests.

Provenance and peer review Not commissioned; externally peer reviewed.

Open Access This is an Open Access article distributed in accordance with the Creative Commons Attribution Non Commercial (CC BY-NC 4.0) license, which permits others to distribute, remix, adapt, build upon this work noncommercially, and license their derivative works on different terms, provided the original work is properly cited and the use is non-commercial. See: http:// creativecommons.org/licenses/by-nc/4.0/

\section{REFERENCES}

1. Johnson NP, Hummelshoj L, World Endometriosis Society Montpellier Consortium. Consensus on current management of endometriosis. Hum Reprod 2013;28:1552-68.

2. Brown J, Farquhar C. An overview of treatments for endometriosis. JAMA 2015;313:296-7.

3. Hirsch M, Duffy JM, Kusznir JO, et al., International Collaboration to Harmonize Outcomes and Measures for Endometriosis. Variation in outcome reporting in endometriosis trials: a systematic review. $A m$ J Obstet Gynecol 2016;214:452-64.

4. Vincent K, Kennedy S, Stratton P. Pain scoring in endometriosis: entry criteria and outcome measures for clinical trials. Report from the Art and Science of Endometriosis meeting. Fertil Steril 2010;93:62-7.

5. Becker CM, Laufer MR, Stratton P, et al., WERF EPHect Working Group. World Endometriosis Research Foundation Endometriosis Phenome and Biobanking Harmonisation Project: I. Surgical phenotype data collection in endometriosis research. Fertil Steril 2014;102:1213-22.

6. Vitonis AF, Vincent K, Rahmioglu N, et al., WERF EPHect Working Group. World Endometriosis Research Foundation Endometriosis Phenome and Biobanking Harmonisation Project: II. Clinical and covariate phenotype data collection in endometriosis research. Fertil Steril 2014;102:1223-32.

7. Rahmioglu N, Fassbinder A, Vitonis AF, et al., WERF EPHect Working Group. World Endometriosis Research Foundation Endometriosis Phenome and Biobanking Harmonization Project: III. Fluid biospecimen collection, processing, and storage in endometriosis research. Fertil Steril 2014;102:1233-43.

8. Fassbinder A, Rahmioglu N, Vitonis AF, et al., WERF EPHect Working Group. World Endometriosis Research Foundation Endometriosis Phenome and Biobanking Harmonisation Project: IV. Tissue collection, processing, and storage in endometriosis research. Fertil Steril 2014;102:1244-53.

9. Williamson PR, Altman DG, Blazeby JM, et al. Developing core outcome sets for clinical trials: issues to consider. Trials 2012;13:132.

10. Khan K. The Crown initiative: journal editors invite researchers to develop Core Outcomes in Women's Health. BJOG 2014;121:1181-2.

11. http://www.comet-initiative.org

12. Dworkin RH, Turk DC, Farrar JT, et al., IMMPACT. Core outcome measures for chronic pain clinical trials: IMMPACT recommendations. Pain 2005;113:9-19.

13. Sinha IP, Smyth RL, Williamson PR. Using the Delphi technique to determine which outcomes to measure in clinical trials: recommendations for the future based on a systematic review of existing studies. PLoS Med 2011;8:e1000393.

14. Egan AM, Smith V, Devane D, et al. Effectiveness of prepregnancy care for women with pregestational diabetes mellitus: protocol for a systematic review of the literature and identification of a core outcomes set using a Delphi survey. Trials 2015;16:356.

15. Guyatt GH, Oxman AD, Kunz R, et al. GRADE guidelines: 2. Framing the question and deciding on important outcomes. $J$ Clin Epidemiol 2011;64:395-400.

16. Boers M, Kirwan JR, Wells G, et al. Developing core outcome measurement sets for clinical trials: OMERACT filter 2.0. J Clin Epidemiol 2014;67:745-53.

17. Prinsen CA, Vohra S, Rose MR, et al. How to select outcome measurement instruments for outcomes included in a "Core Outcome Set"-a practical guideline. Trials 2016;17:449.

18. Chan AW, Tetzlaff JM, Altman DG, et al. SPIRIT 2013 Statement defining standard protocol items for clinical trials. Ann Intern Med 2013;158:200-7. 